\title{
Enantioselective Photochemical Synthesis of a Simple Alkene via the Solid State Ionic Chiral Auxiliary Approach
}

\author{
Shuang Chen, Brian O. Patrick, and John R. Scheffer*
}

Department of Chemistry, University of British Columbia, 2036 Main Mall, Vancouver, B.C., Canada V6T 1Z1

Scheffer@chem.ubc.ca

Supporting Information

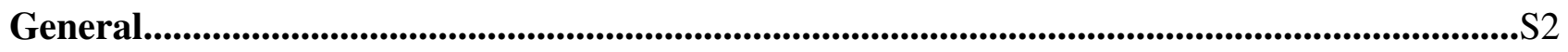

(3a $\alpha, 7 \mathbf{a} \alpha)$-2-(Bromomethyl)-2,3,3a,4,7,7a-hexahydro-1H-indene (10)...........................................S3

Methyl 4-\{2-[(3a $\alpha, 7 \mathbf{a} \alpha)-2,3,3 a, 4,7,7 a-h e x a h y d r o-1 H$-inden-2-yl]-

1-hydroxyethyl\} benzoate (11)...........................................................................................................................S4

Methyl 4-[(2 $\alpha, 3 \mathbf{a} \alpha, 7 \mathbf{a} \alpha)$-octahydro-1 $H$-inden-2-ylacetyl]benzoate (1a)..........................................S5

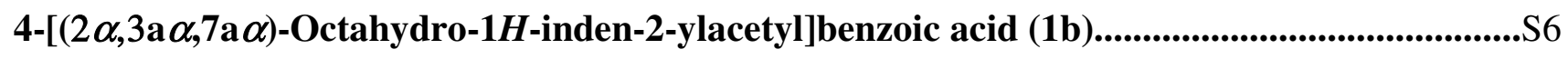

L-Prolinamide Salt of Keto-acid 1b (1c)............................................................................................

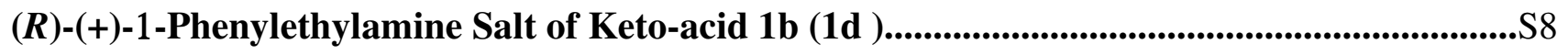

(S)-(-)-1-Phenylethylamine Salt of Keto-acid 1b (1e)..........................................................................S9

$(1 R, 2 S)$-(+)-cis-1-Amino-2-indanol Salt of Keto-acid 1b (1f)...........................................................S10

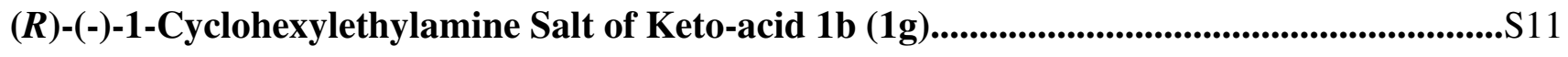

$(1 \mathrm{a} \alpha, \mathbf{1 b} \alpha, 5 \mathrm{a} \alpha, \mathbf{6 a} \alpha)$-Octahydro-1aH-indeno[1,2-b] oxirene (7),.......................................................S12 


\section{General Information}

Commercial spectral grade solvents were used for photochemical experiments unless otherwise stated. For synthetic use tetrahydrofuran and diethyl ether were dried over sodium/benzophenone complex; dichloromethane was dried over calcium hydride; acetonitrile was distilled from $\mathrm{P}_{2} \mathrm{O}_{5}$. All reactions were performed under nitrogen atmosphere unless otherwise noted. Melting points were determined on a Fisher-Johns hot-stage apparatus and are uncorrected. Analytical TLC was performed on $0.20 \mathrm{~mm}$ silica gel $60-\mathrm{F}$ plates and visualized under UV light and/or by staining with $\mathrm{I}_{2}$ or phosphomolybdic acid. Column chromatography was performed using 230-400 mesh silica gel slurry packed with the eluting solvent. ${ }^{1} \mathrm{H}$ NMR spectra were obtained at either 300 or $400 \mathrm{MHz}$, as indicated. ${ }^{13} \mathrm{C}$ NMR spectra were recorded at either $75 \mathrm{MHz}$ or $100 \mathrm{MHz}$. UV spectra were measured at $25^{\circ} \mathrm{C}$ in methanol. The enantiomeric purity of epoxide 7 was determined via chiral GC (SUPELCO $\beta$ $\operatorname{Dex}^{\mathrm{TM}} 350$ Custom Capillary Column, $120^{\circ} \mathrm{C}$ at $\left.1.37 \mathrm{~mL} / \mathrm{min}\right)$. 


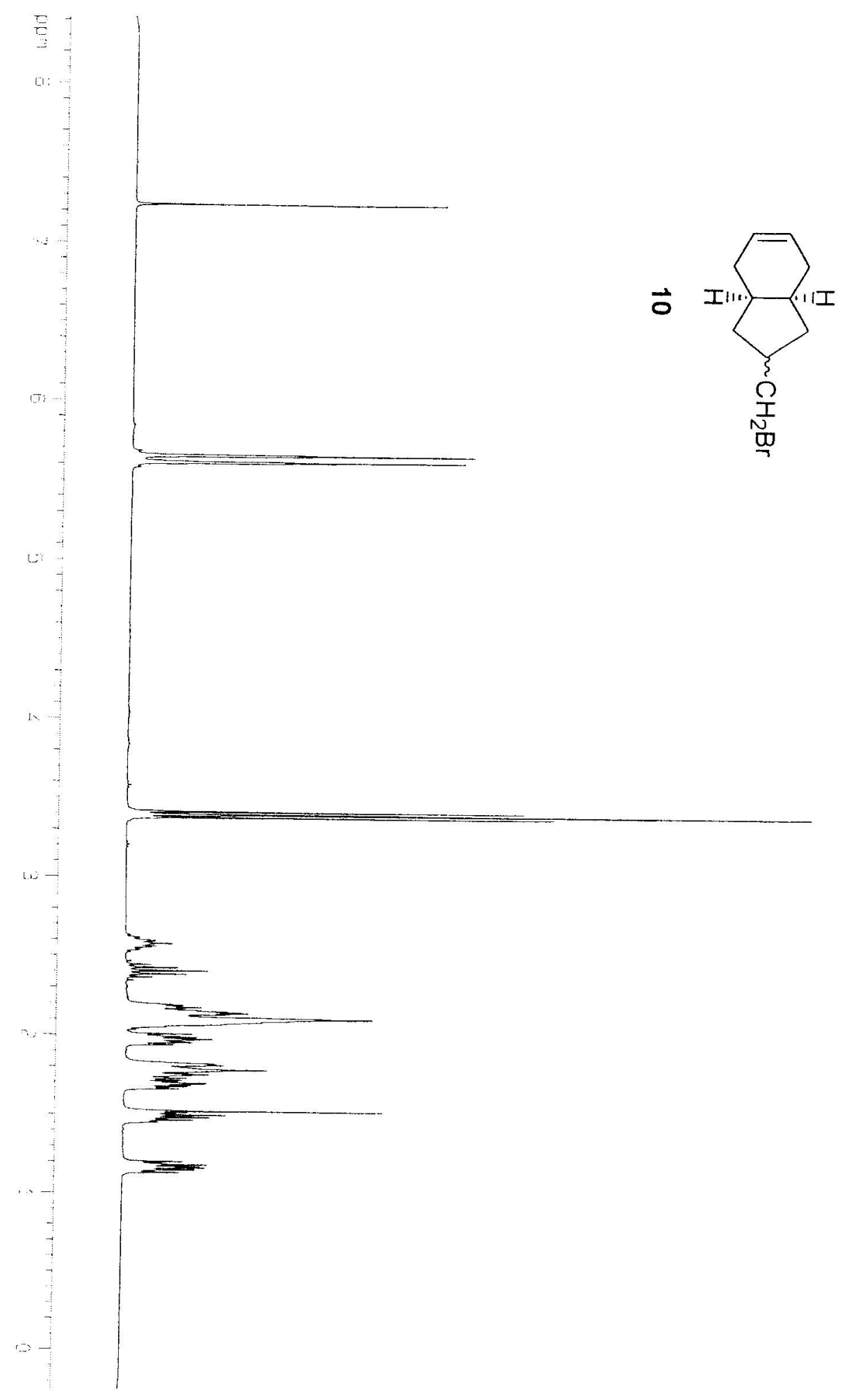

S3 


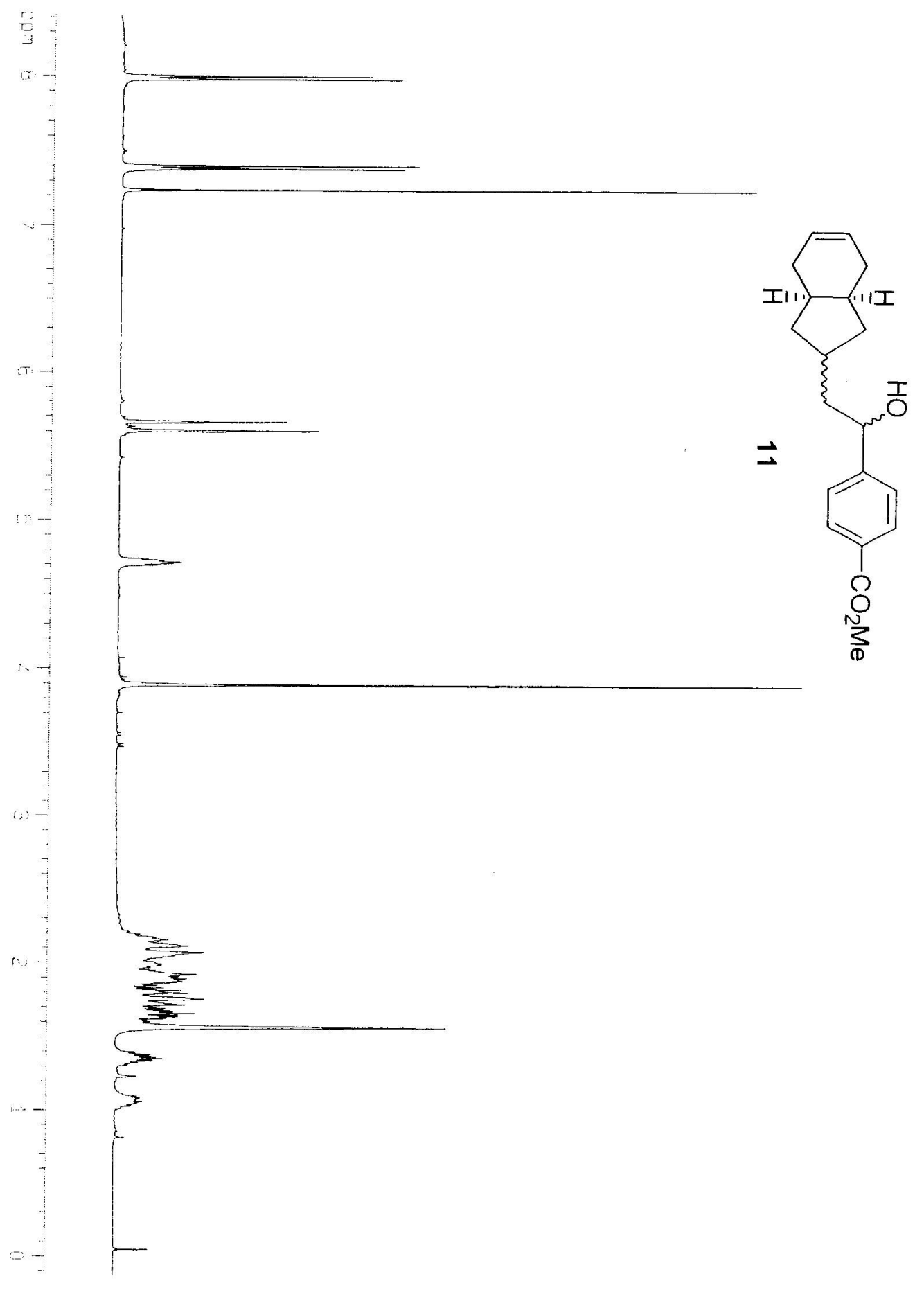




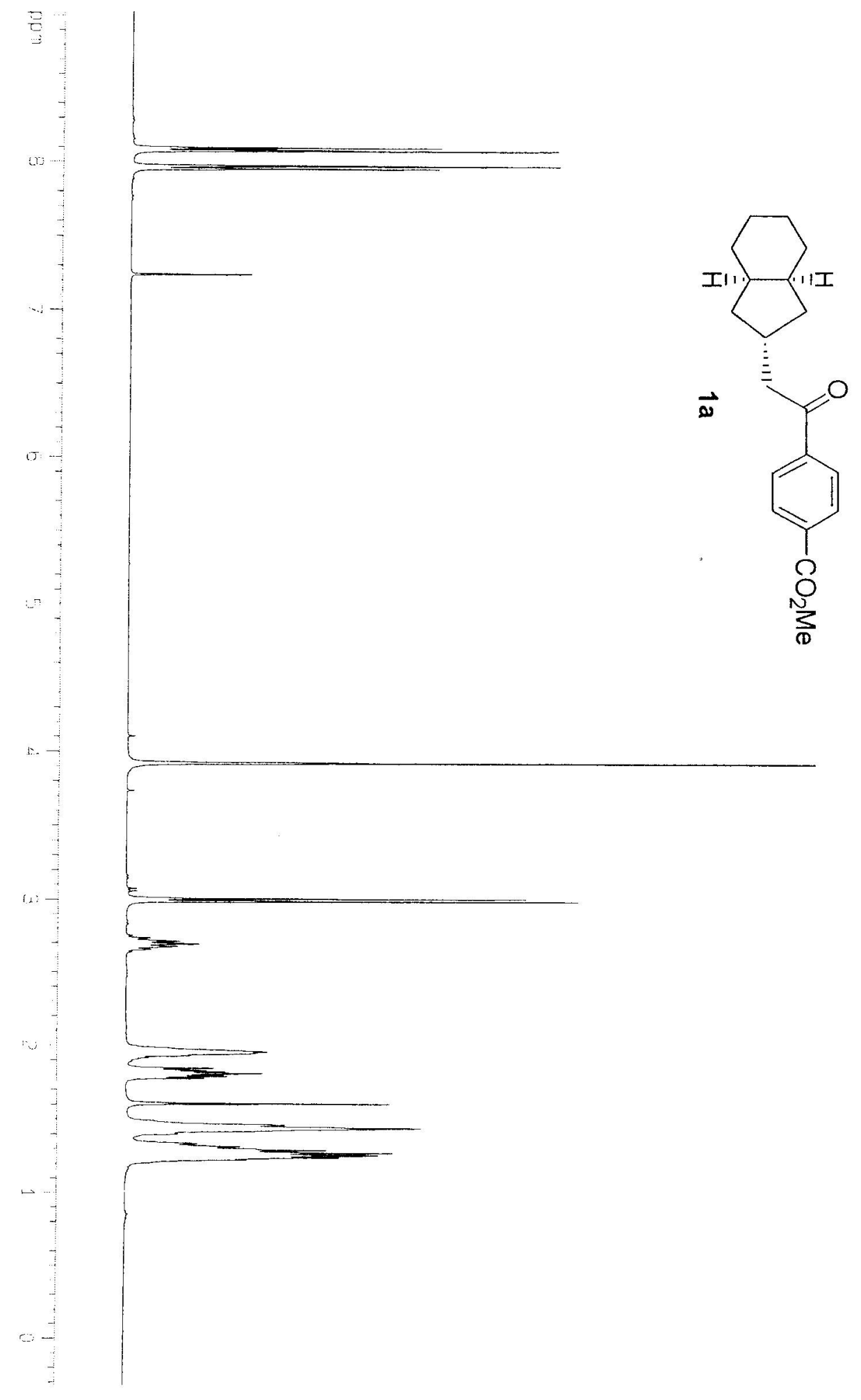




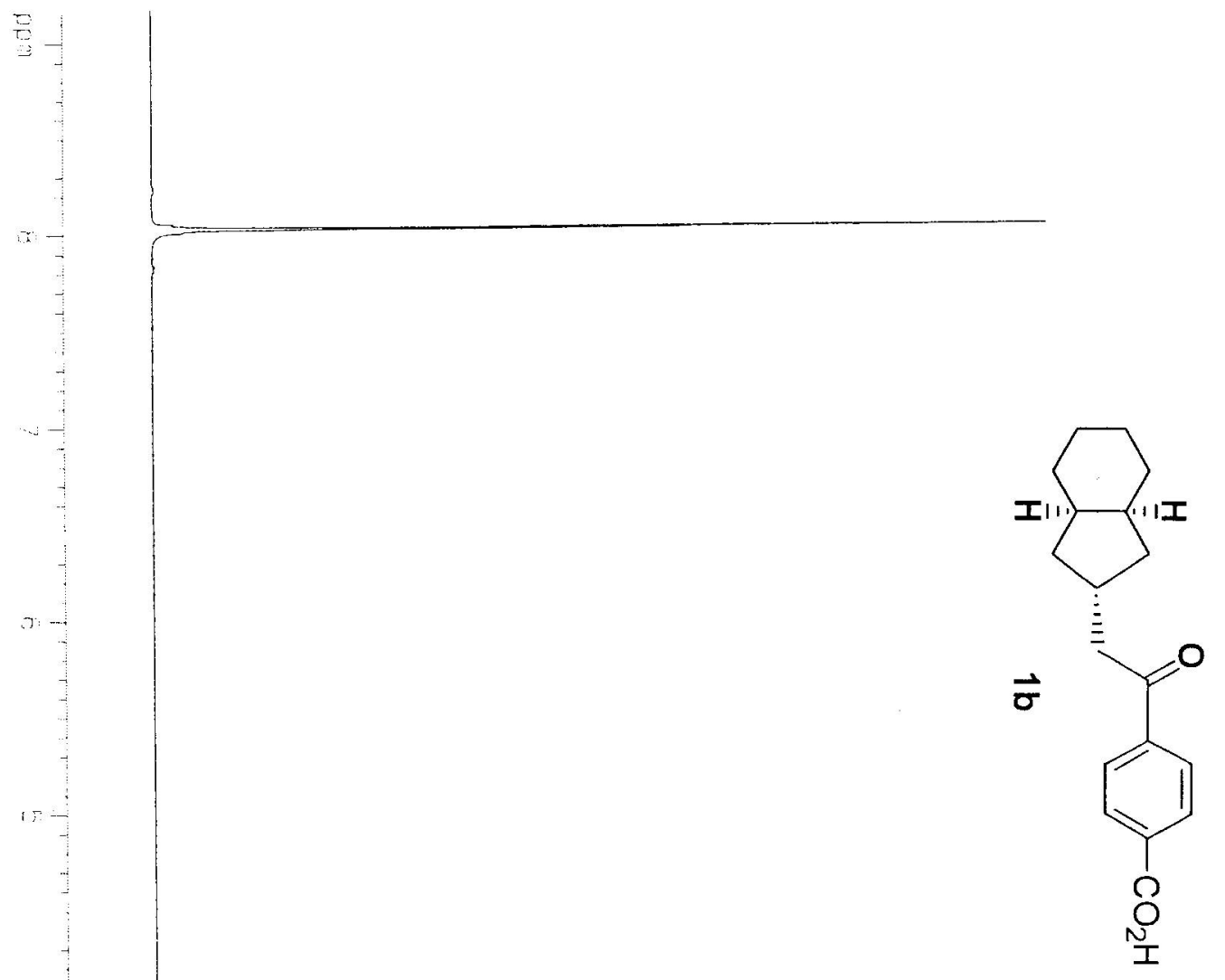




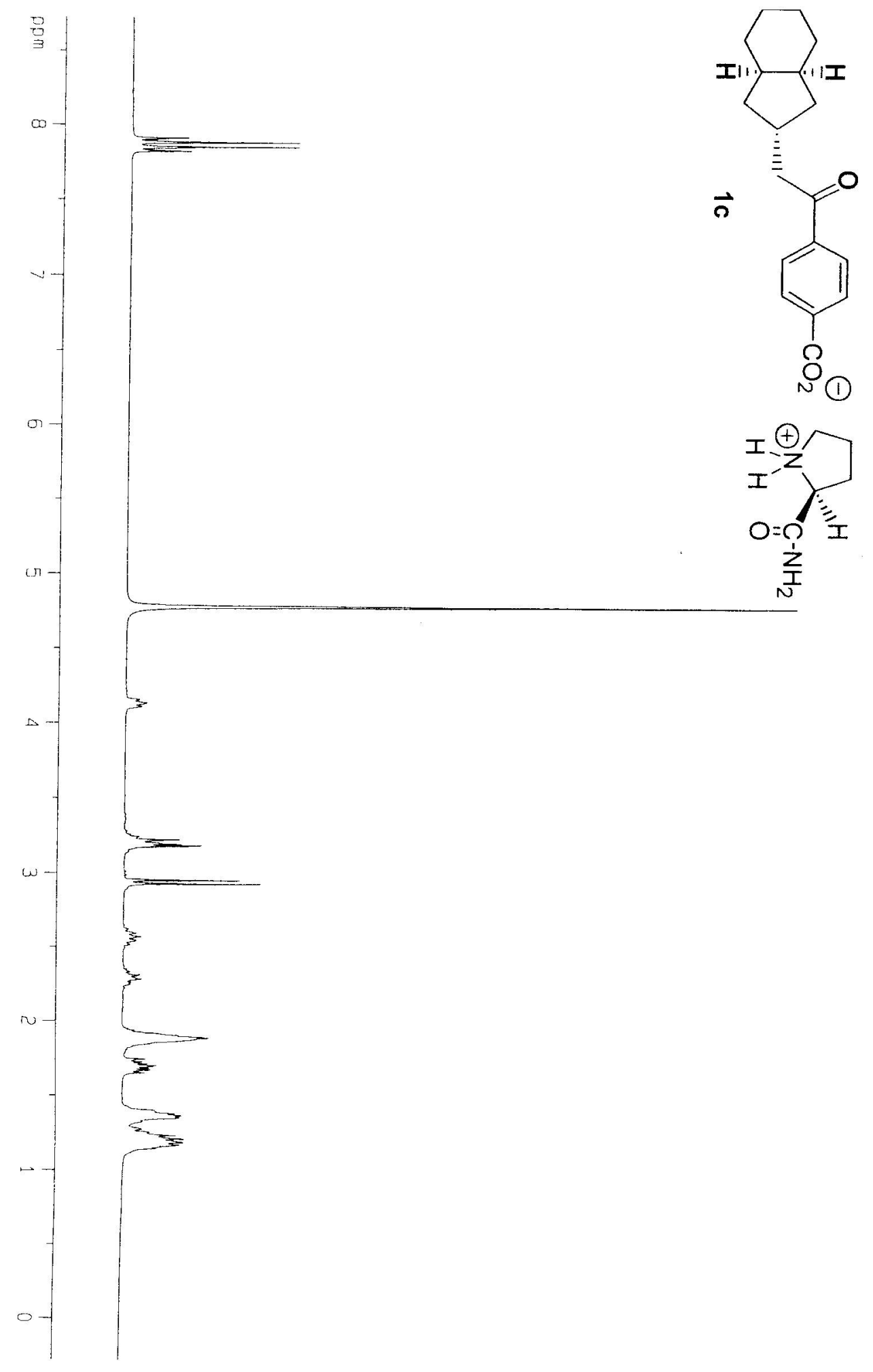




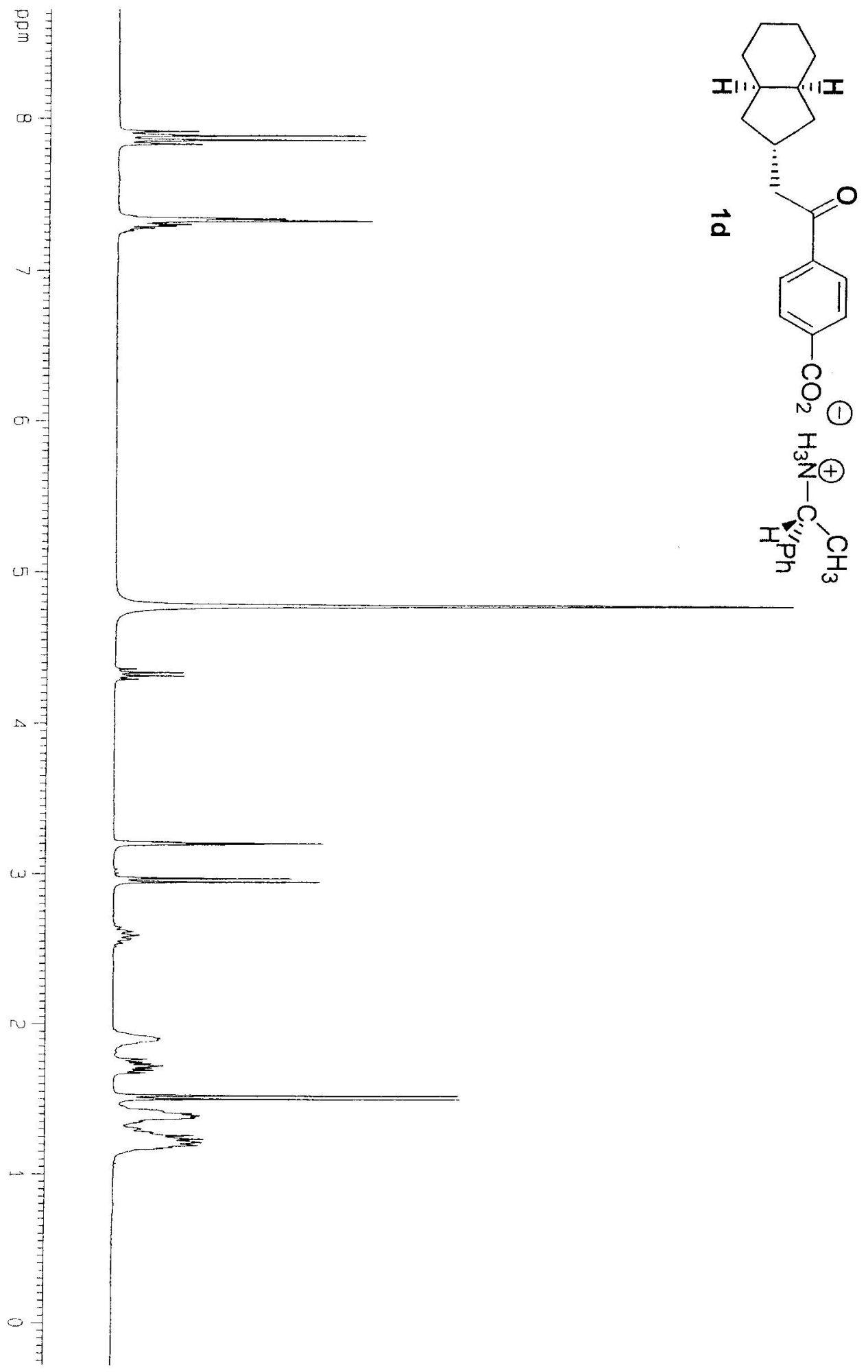




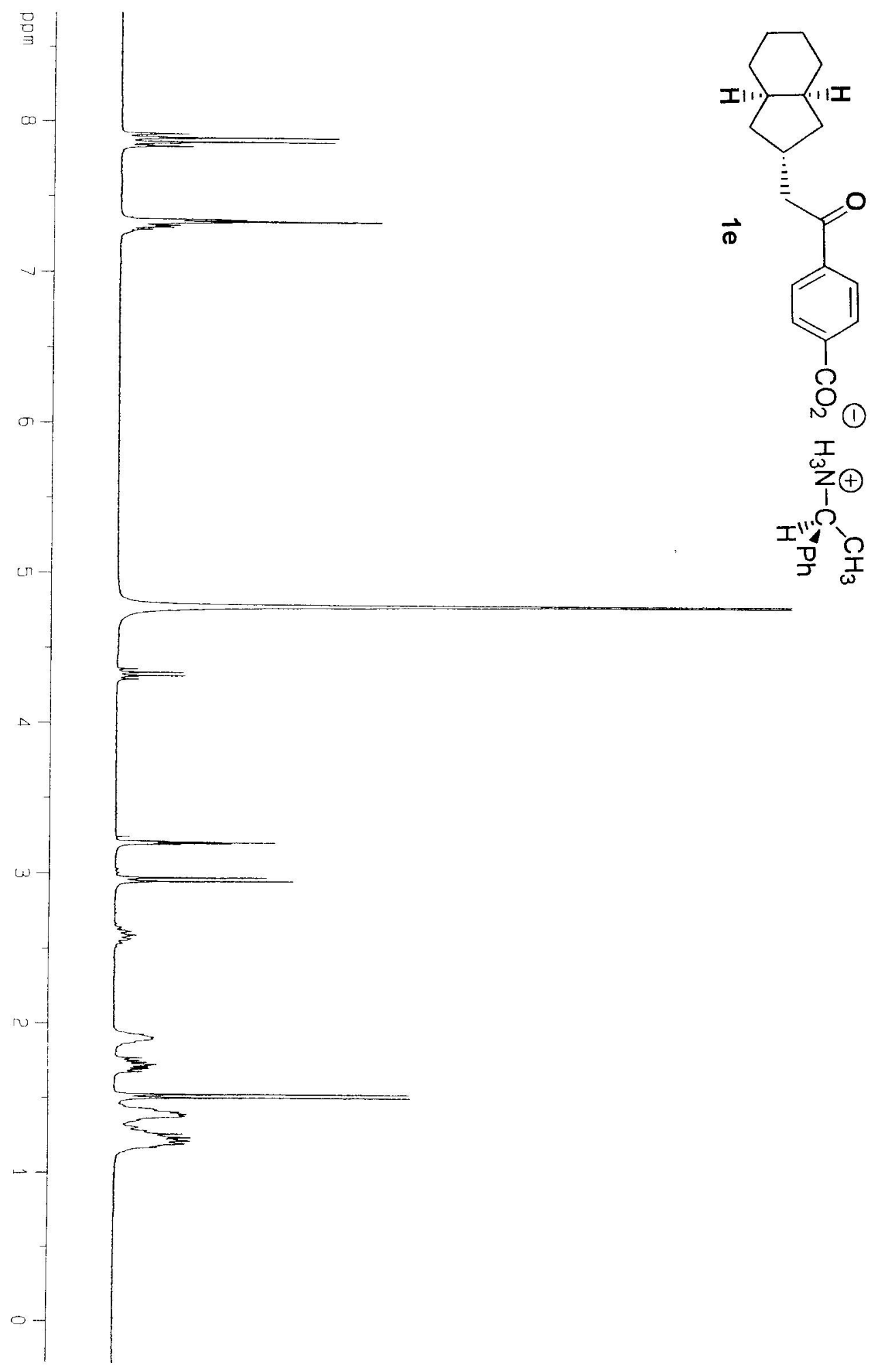

S9 


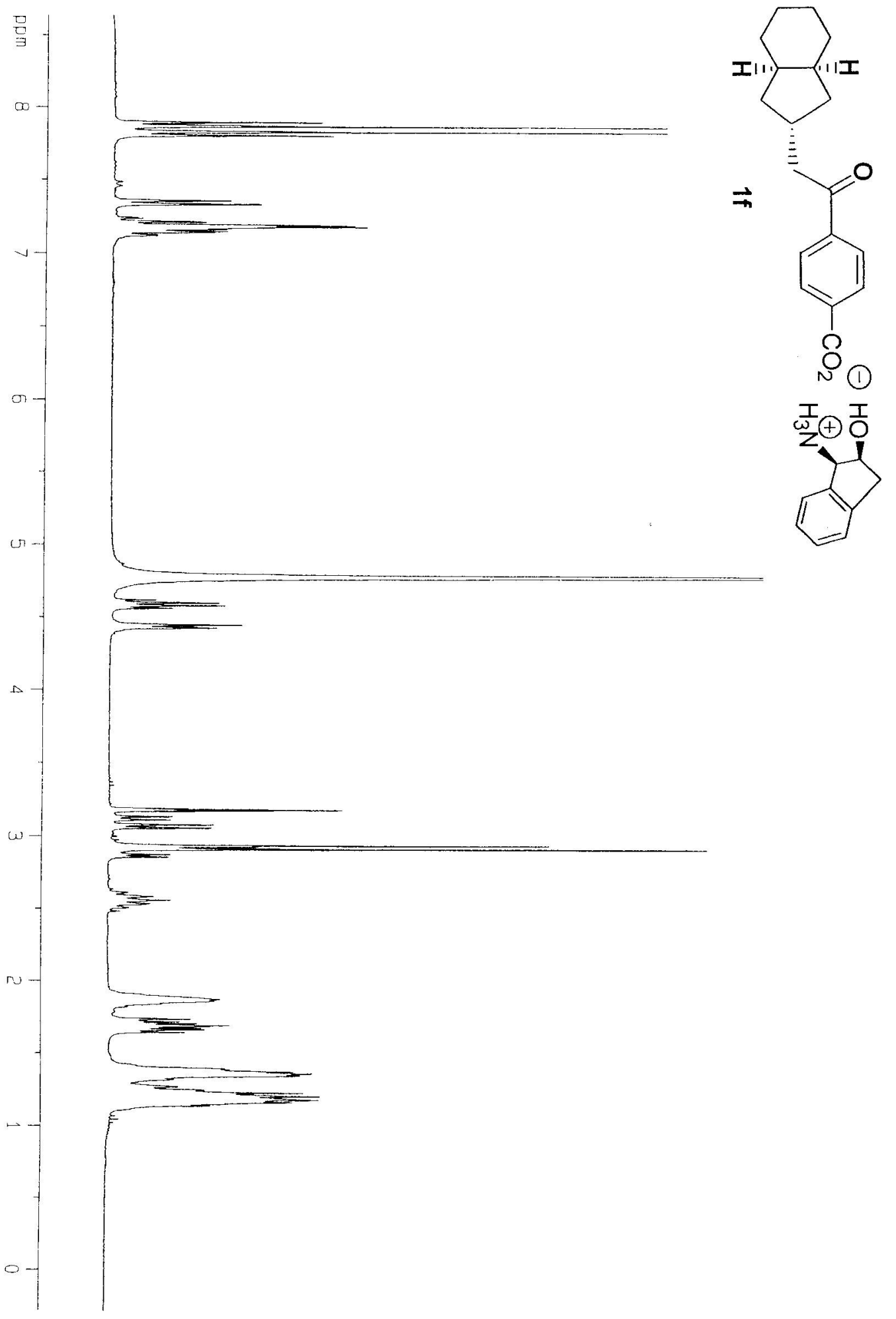




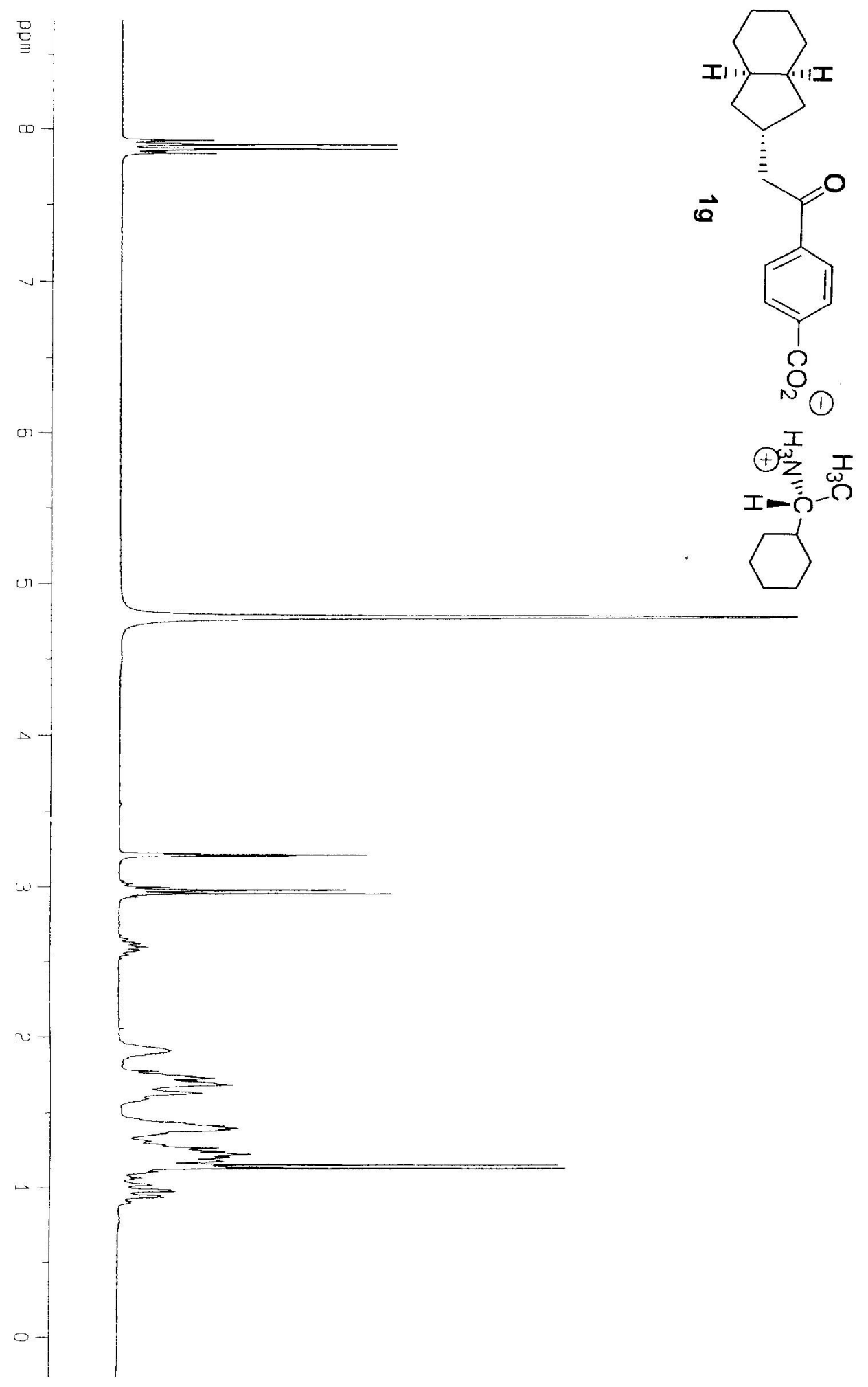




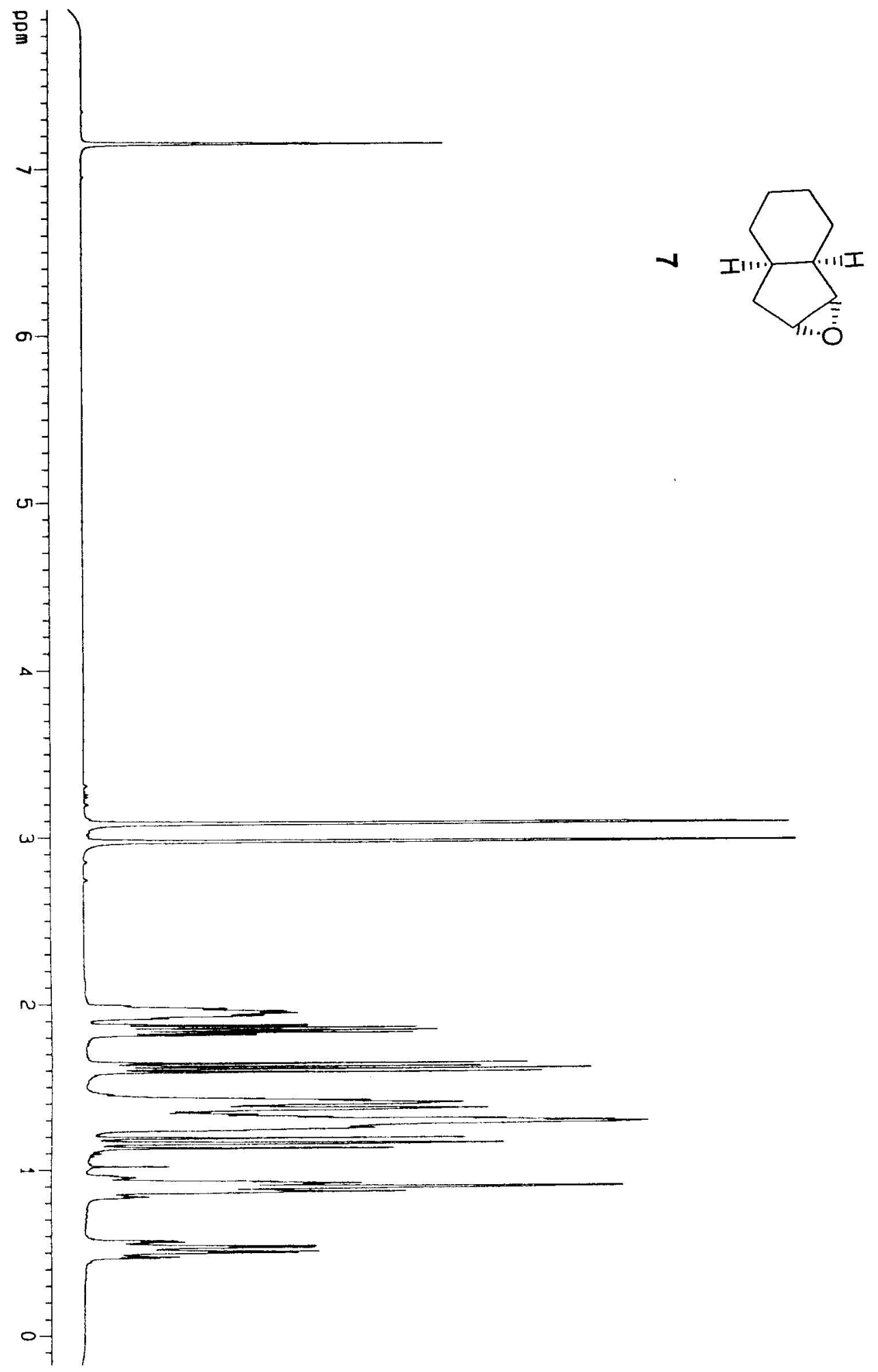

